\title{
OBSERVATIONS OF MR FLUID CLUMPING BEHAVIOUR IN A SQUEEZE-MODE DAMPER
}

\author{
Bogdan SAPIŃSKI \\ *Department of Process Control, AGH University of Science and Technology, Al. Mickiewicza 30, 30-059 Kraków, Poland \\ deep@agh.edu.pl
}

received 25 February 2016, revised 25 July 2016, accepted 27 July 2016

\begin{abstract}
The study summarises the results of quasi-static experimental tests of an MR squeeze-mode damper prototype (MRSQD) performed at the MTS testing machine. Of particular interests was the influence of MR fluid clumping behaviour in the MRSQD working gap on the force output of the device. The MRSQD tests were assessed by measuring the damping force output at prescribed sinusoidal displacement inputs and at various (fixed) voltage levels resulting in the respective average current levels in the control coil. The influence of piston position offset on the damping force was also investigated. The collected data were shown in the form of damping force time histories and damping force-piston displacements loops and discussed with respect to MR fluid clumping behaviour.
\end{abstract}

Key words: MR Fluid, Squeeze Mode, Damper, Clumping Behaviour

\section{INTRODUCTION}

In consideration of the manner in which the material is utilized in a controllable device, MR fluid-based hardware is separated into following categories: flow (valve) mode, direct shear (clutch) mode, squeeze mode and gradient pinch mode. To date only the flow mode- and shear mode devices have been commercialized. No squeeze mode-hardware has been reported beyond the academia so far with the exception given in (Kim, 2014).

In squeeze mode-devices the flow channel height varies in the direction parallel to that of the magnetic field. As the distance between the opposing surfaces changes, the MR fluid is squeezed out and into the flow channel (Fig.1). In this mode, MR fluids can generate a large range of controllable force in envelopes that can only accommodate small strokes - stresses approach $100-150 \mathrm{kPa}$ with displacements of the order of several millimeters. This fact indicates the potential applications of MR fluids.

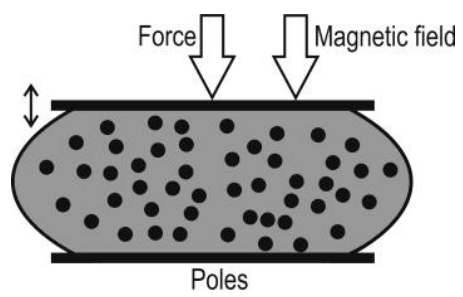

Fig. 1. MR fluid in squeeze mode

A very interesting aspect of the squeeze mode is the clumping phenomenon. How to minimise the effect of clumping behaviour is still a challenging problem. Research data reported so far reveal that if the same test is repeated without mixing the MR fluid, a progressively increasing force will be developed in consecutive test runs. This behaviour is suspected to be due to particle aggre- gation in MR fluid during squeeze process. As the MR fluid is to be squeezed, iron particles are trapped in the magnetic field in the gap while the carrier fluid is radially flowing outward. This causes iron particles to form aggregates in the gap and separate from the carrier fluid, and this effect is intensified after each test repeat (see Fig. 2).

Farjud et al. (2011a) proposed the definition to quantify clumping behaviour and revealed that the effect of clumping can be greatly reduced by a magnetic dither during piston return that can help the clumped particles re-disperse in the MR fluid. The reduction of clumping effect is because during piston return the dither can cause the iron particles to escape aggregates and for new chains.

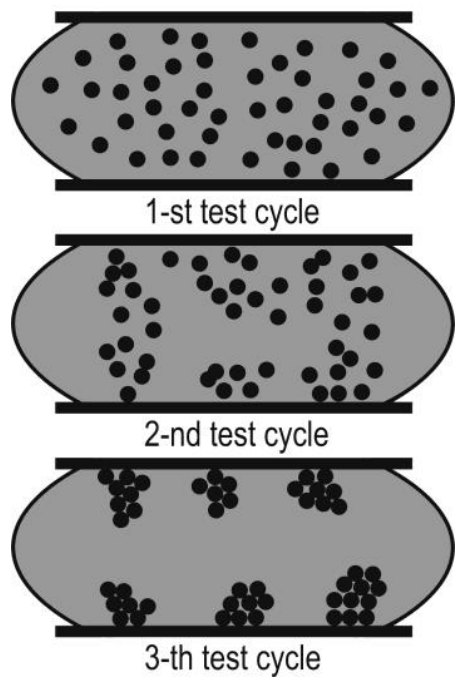

Fig. 2. Illustration of MR fluid clumping behaviour

The flow and direct shear modes abound in reports in the literature whilst there is little published data relating to the squeeze 
mode. As regards the pinch mode, there are reports of few studies. Because many properties of MR fluids in the squeeze mode are still unknown, this mode has recently received recently a great deal of attention and still poses a major challenge to researchers. The studies exploring MR fluids in the squeeze mode and the involved phenomena are but a few. For example, (Mazlan et al., 2007; Farjoud at al., 2009a, b) revealed that MR fluids can provide a much larger range of controllable force in small operational envelopes compared to flow and direct shear modes. Systematic investigation of MR fluids reported in squeeze mode research described in (Mazlan et al., 2012) showed that the fluids experienced rheological changes in three stages during compression and tension and that fluid-particles separation phenomenon was the main cause of the unique behaviour of MR fluids. (Horak, 2013) showed that in the presence of a magnetic field, iron particles are arranged in column-like chain structures which get deformed due to compression and the MR fluid provides increased resistance to compressive loading and its yield stress tends to increase. Guo et al., (2013) studied compression behaviour of MR fluids under nonuniform magnetic field and showed that there exists a magnetic-field gradient-induced normal force when compared to the uniform field.

As regards the MR squeeze-mode dampers, one of the first major contribution was an MR semi-active damper (Jolly et al., 1996). Another attempt was a dual-coil mixed-mode damper which utilized shear and squeeze mode (Manecke et al., 2006). (Mazlan, 2008) examined the behavior of water-based MR fluids and hydrocarbon oil based MR fluids under compressive and tensile loads. (Farjoud et al., 2009) tested a squeeze-mode rheometer and provided a non-dimensional model which was validated experimentally. The same author(s) revealed a squeeze-mode damper prototype and developed a mathematical model of the damper suitable for design and engineering studies (Farjoud et al., 2011a; Farjoud, 2011b; Zhang et al., 2011). Gołdasz and Sapiński (2011) provided a numerical study in which a squeeze-mode mount was subjected to sweep-sine displacement inputs and characterized the mount model in terms of dynamic stiffness and damping vs. frequency. Gong et al. (2014) tested a dual-cavity high-force damper subjected to sinusoidal displacement inputs and fixed coil currents. The most recent study (Sapiński and Gołdasz, 2015) summarises the results of an MR damper prototype development and performance evaluation study.

The objective of the study was to analyse MR fluid clumping behaviour in the MRSQD in quasi-static states. For this purpose the device was tested in the MTS testing machine under predetermined sinusoidal displacement inputs and at various (fixed) voltage levels resulting in the respective average current levels in the control coil. Influence of piston position offset on the damping force was also investigated. The MR fluid clumping behaviour was discussed basing on the determined performance figures of the MRSQD (force time histories and force-piston displacement loops).

The paper is organized as follows. Section 1 outlines state-ofthe art. solutions in the field of squeeze mode of MR fluids, MR fluid-based hardware and clumping phenomenon of MR fluids. Section 2 summarises the structure and operation principle of the MRSQD. Section 3 discusses experimental results of the device in the context of MR fluid clumping behaviour. The final conclusions are given in Section 4.

\section{MR SQUEEZE-MODE DAMPER}

A schematic diagram of the MRSQD is shown Fig. 3. The numeric symbols in the figure indicate all key components of the MRSQD (1-8) and the key highlights materials used for manufacturing of the device. The hardware features two concentric cylinders $(1,2)$. The inner non-magnetic cylinder (2) houses the piston (3), the core assembly (4), and the floating piston (5). The core assembly incorporates the coil (6). The outer cylinder (1) is made of a ferromagnetic material. The distance between the lower surface of the piston and the upper surface of the core is referred to as the working gap of time-varying height. The distance between the piston and the core varies according to the prescribed displacement (force) input (along the vertical z-axis). The floating piston below the core assembly separates the MR fluid from the coil spring located in the compensating chamber below the floating piston (5). The chamber incorporates a preloaded spring system (not shown in Fig. 2) for fluid volume compensation. Alternatively, the compensating chamber could be filled in with pressurized gas. The choice of the coil spring was due to convenience. The current in the control coil induces a magnetic field of the strength $H$. The magnetic flux generated by the current in the control coil (6) travels through the core and into the control gap, the outer cylinder, and back into the core through radially projected arms in the core base. The inner cylinder (2) of sufficient wall thickness is used to reduce the amount of magnetic flux bypassing the control gap, i.e. magnetic short circuit. All of the components ensure an efficient magnetic flux return path.

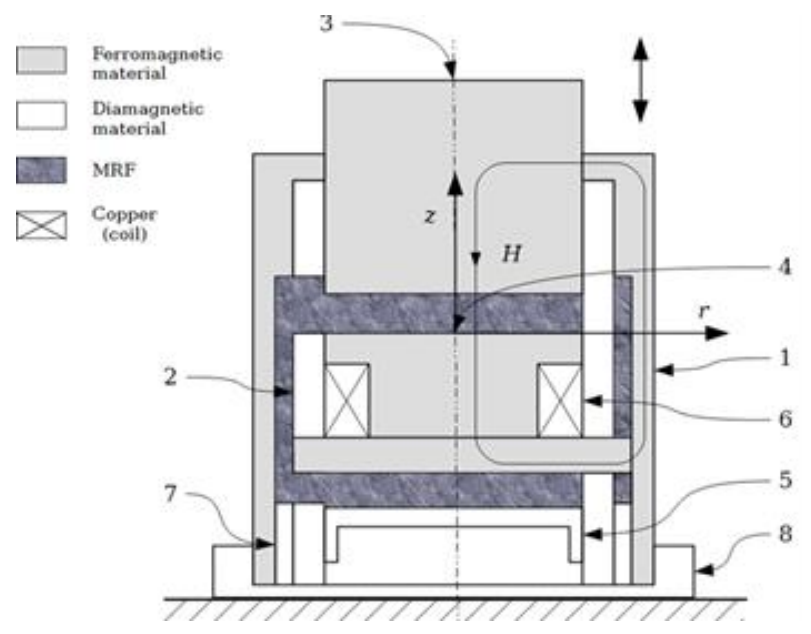

Fig. 3. Schematic diagram of the MRSQD

The flux induced in the working gap upon the application of the coil current effectively modifies the yield stress of the fluid, and its resistance to flow. As the piston moves downward, the distance between the core and the piston decreases. The excess of MR fluid is squeezed out of the working gap into the fluid volume between the inner cylinder and the outer housing of the MRSQD, and then into the compensating chamber. The additional fluid volume that enters the compensating chamber pushes the floating piston against the coil spring. The structure incorporates a non-magnetic ring (7), whereas the base cap (8) is used for fixing the assembly against the ground. The outer cylinder length is $133 \mathrm{~mm}$ and the diameter is $102 \mathrm{~mm}$. The MR fluid type employed in the MRSQD is BASF Basonetic 4035 (Kieburg, 2010) with the volume of $110 \mathrm{ml}$. 


\section{EXPERIMENTS}

The MRSQD was tested in a MTS 810 machine driven by a FlexTest SE type controller (Fig. 4). The displacement of the piston was measured using a LVDT type sensor, whereas the force output of was read out by the force sensor).

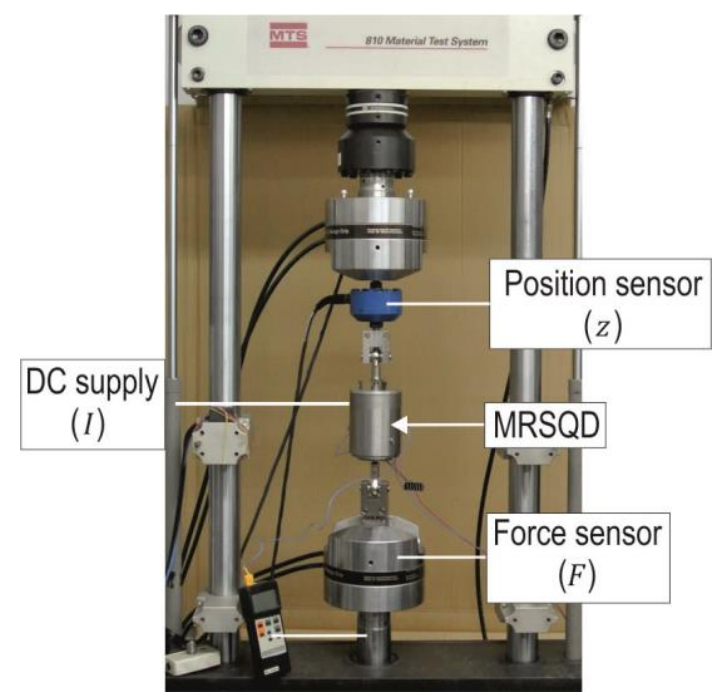

Fig. 4. MRSQD ready for tests

Experimental tests were performed to investigate the influence of the parameters of the sine piston displacement input (amplitude $A$ and frequency $f$ ), the current level in the control coil Iand piston offset $x_{0}$ on the force generated by the device and to obtain the force vs time and force vs force displacement plots. The experiment involved two stages. In the first stage the piston was displaced with respect to its midpoint position $x_{0}=0 \mathrm{~mm}$, and in the stage - with respect to the piston position shifted downwards $x_{0}=1.44 \mathrm{~mm}$ (Fig. 5).

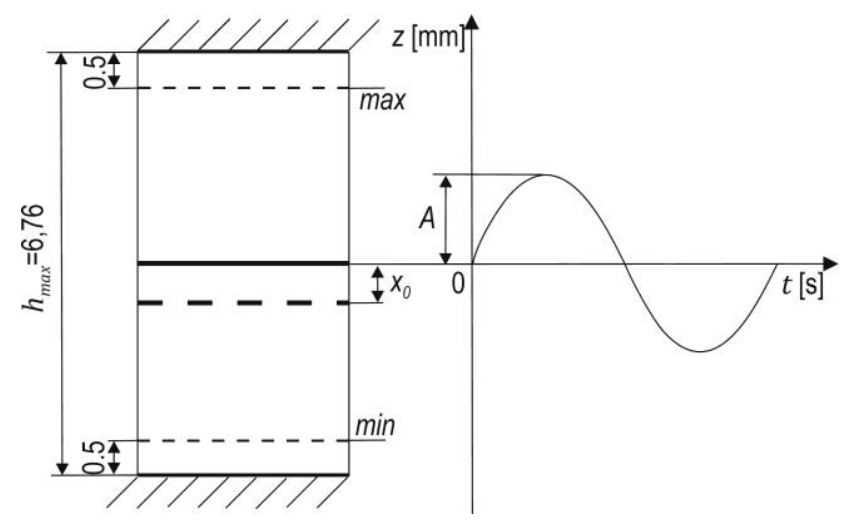

Fig. 5. Illustration of sinusoidal displacement of the piston in the gap

The device testing was assessed by measuring the force output at prescribed sinusoidal displacement inputs $x(t)=x_{0}+A \cdot \sin (2 \pi f t)$ at various (fixed) voltage levels resulting in the average coil current level $I: 0,1,2$ and $3 \mathrm{~A}$, respectively. For the purpose of experiments the data were collected within the range of frequency $f(1,10) \mathrm{Hz}$ with the step $1 \mathrm{~Hz}$ and for the following displacement amplitude $A: 0.72$, 1.44 and $2.88 \mathrm{~mm}$, respectively. The effect of piston position offset on the damping force waveform was investigated, too. In the tested MRSQD the maximum gap height $h_{\max }$ was equal to $6.76 \mathrm{~mm}$, and displacement input amplitude range was limited up to $A=2.88 \mathrm{~mm}$ in order to preserve a minimum gap of $0.5 \mathrm{~mm}$ on either side of the piston, i.e. between the lower surface of the piston and the upper surface of the core on one side and between the piston and the stop position on the other. Throughout the tests at room temperature of $25^{\circ} \mathrm{C}$ the signal sampling frequency was $1 \mathrm{kHz}$.

Selected tests results are depicted in Fig. 6-11, plotting force time histories in the range $(1,10) \mathrm{s}$ and force-displacement loops registered in 2-nd test cycle, and 8-th test cycle of the piston motion. In Figs 6-9 $x_{0}=0 \mathrm{~mm}$ while in Fig. 10 and $11, x_{0}=1.44 \mathrm{~mm}$. Plots in Fig. 6 and 7 compare force time histories and force-displacement loops for current level $1 \mathrm{~A}$ and $3 \mathrm{~A}$, and displacement input with amplitude $A=2.88 \mathrm{~mm}$, and frequency $1 \mathrm{~Hz}$ and $4 \mathrm{~Hz}$. It is apparent that the when the current is higher, the damping force gets higher, too. It should be noted that critical value of force measured by the force sensor was adjusted to be $5000 \mathrm{~N}$. Since the damping force reaches this value in the 4-th test cycle, the force-displacement loop in this case is represented by a "point" with force value of about $3900 \mathrm{~N}$ and position of $z=2.88 \mathrm{~mm}$. In this case the minimal height of the squeezed layer of MR fluid is $0.5 \mathrm{~mm}$. A similar tendency is revealed in plots shown in Fig. 8 and 9, though in these case the damping force value $5000 \mathrm{~N}$ is not exceeded. This is so because the amplitude is $A=1.44 \mathrm{~mm}$, i.e. two times smaller. In this case the minimal height of the squeezed layer of MR fluid is $1.94 \mathrm{~mm}$. It appears that for such piston displacement amplitude and current levels in the control coils, the influence of frequency on the damping force is rather minor. Plots in Fig. 10 and 11 present force time histories and forcedisplacement loops at piston position offset $x_{0}=1.44 \mathrm{~mm}$ for current level $1 \mathrm{~A}$ and $3 \mathrm{~A}$, displacement inputs with amplitude $A=1.44 \mathrm{~mm}$, frequency $1 \mathrm{~Hz}$ and $4 \mathrm{~Hz}$. Note that in this case, the amplitude of displacement is the same as in case shown in Fig. 8 and 9. Comparing the plots in those figures, it is apparent that the influence of the piston position offset on the damper force is rather minor. Of particular importance are force-displacement loops in Fig. 10b. The actual shape of these plots is associated with the restriction of the damping force measured by the force sensor (similar case to that depicted in Fig. 6b, see the plot of the 8-th test cycle).

The presented plots clearly reveal a lack of symmetry - the force values are lower when the piston moves upwards than during its downward movement. When analysing force time histories in each test an increase of the damping force is observed with each cycle of the piston movement. This is caused by the clumping behavior of MR fluid in the working gap. When comparing the plots through Fig 6a, 8a to $10 \mathrm{a}$, it is apparent that at the assumed current level in the control coil $(F 1 \mathrm{~A})$, the lower height of MR fluid squeezed, the higher damping force. Moreover, in the assumed time range the damping force does not reach steady state value. Similar situation refers to the plots in Fig. $6 \mathrm{~b}$ and $8 \mathrm{~b}$ (then $F 3 \mathrm{~A}$ ) but then the damping force reaches higher values due to the higher level of the current in the control coil. The plots in Fig. 7a and 9a show that at the assumed frequency displacement input ( $f=4 \mathrm{~Hz}$ ), the damping force achieves steady state value the contrary to the situation presented in Fig. 6a and 8a (then $f=1 \mathrm{~Hz}$ ). 

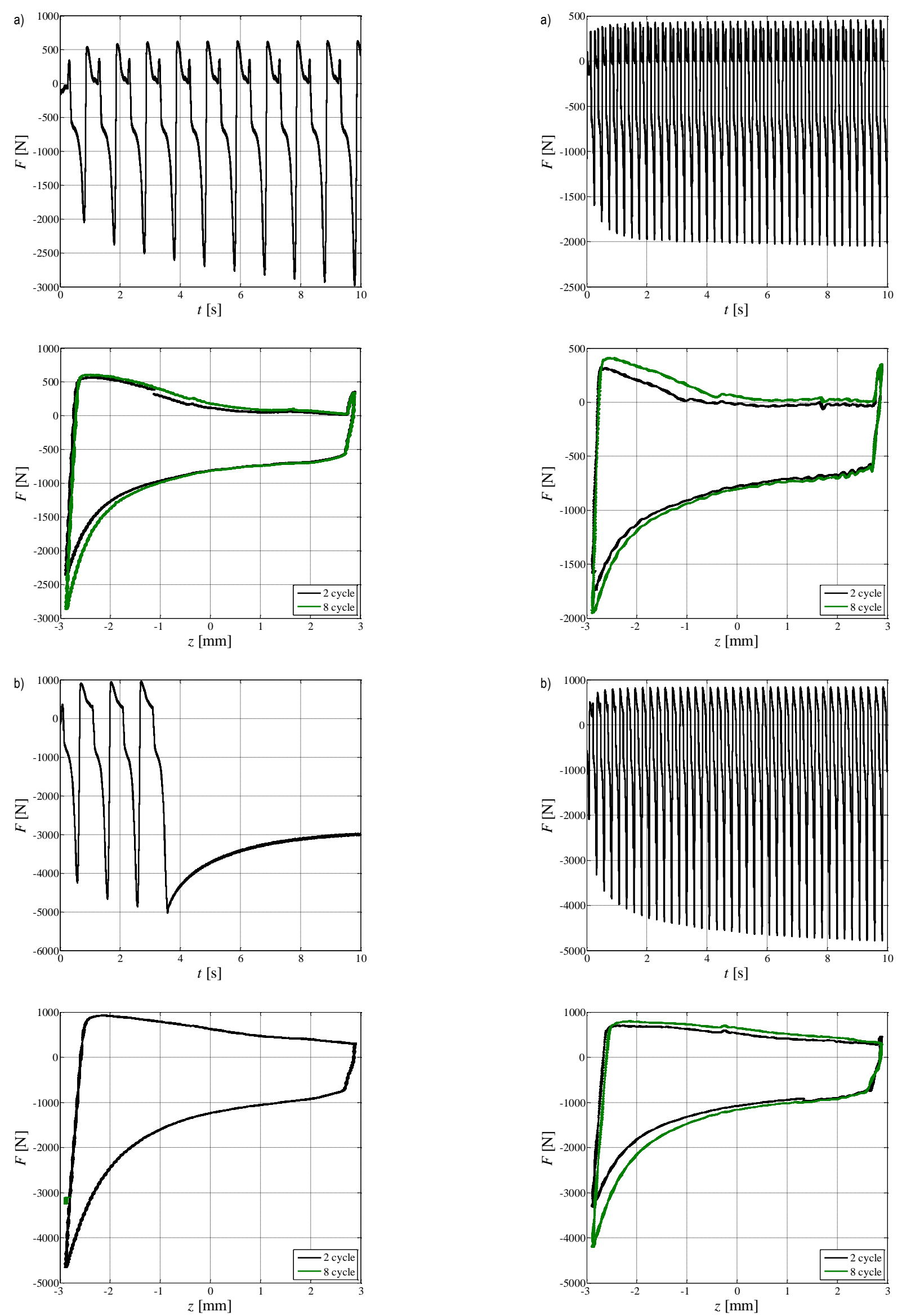

Fig. 6. Force vs. time and force vs. piston displacement; $A=2.88 \mathrm{~mm}$, $f=1 \mathrm{~Hz}$ : a) $l=1.0 \mathrm{~A}$, b) $l=3.0 \mathrm{~A}$

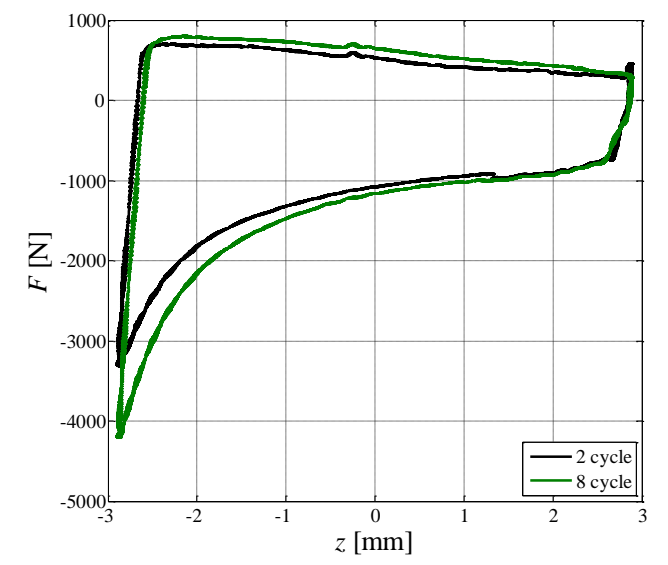

Fig. 7. Force vs. time and force vs. piston displacement; $A=2.88 \mathrm{~mm}$, $f=4 \mathrm{~Hz}$ : a) $l=1.0 \mathrm{~A}$, b) $l=3.0 \mathrm{~A}$ 
a)
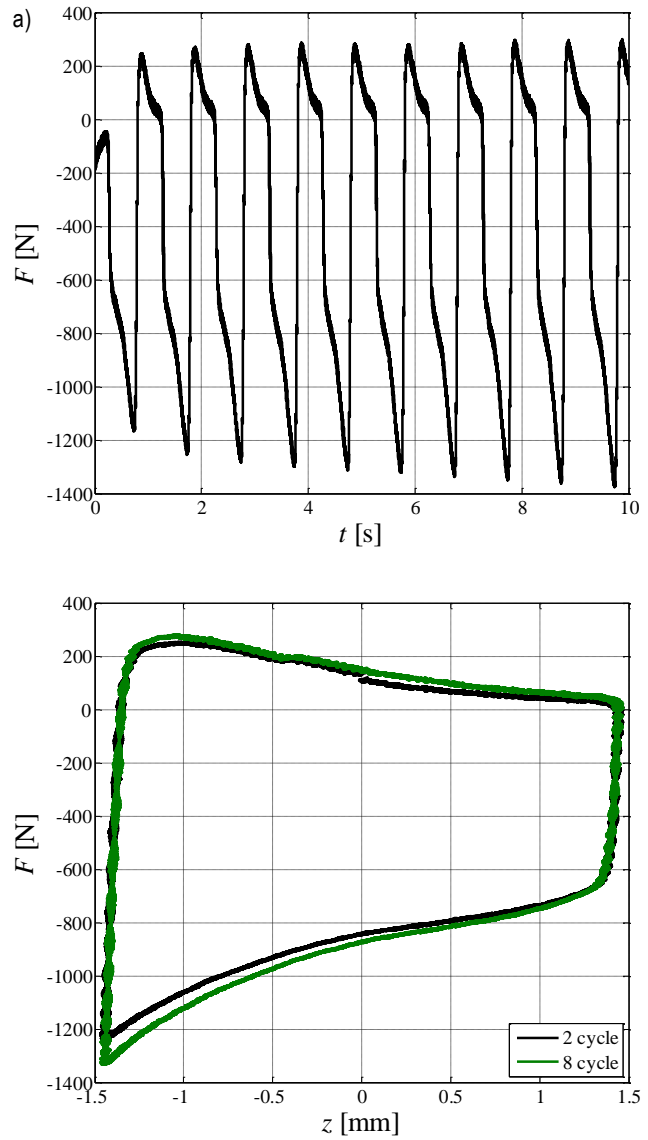

b) 1000
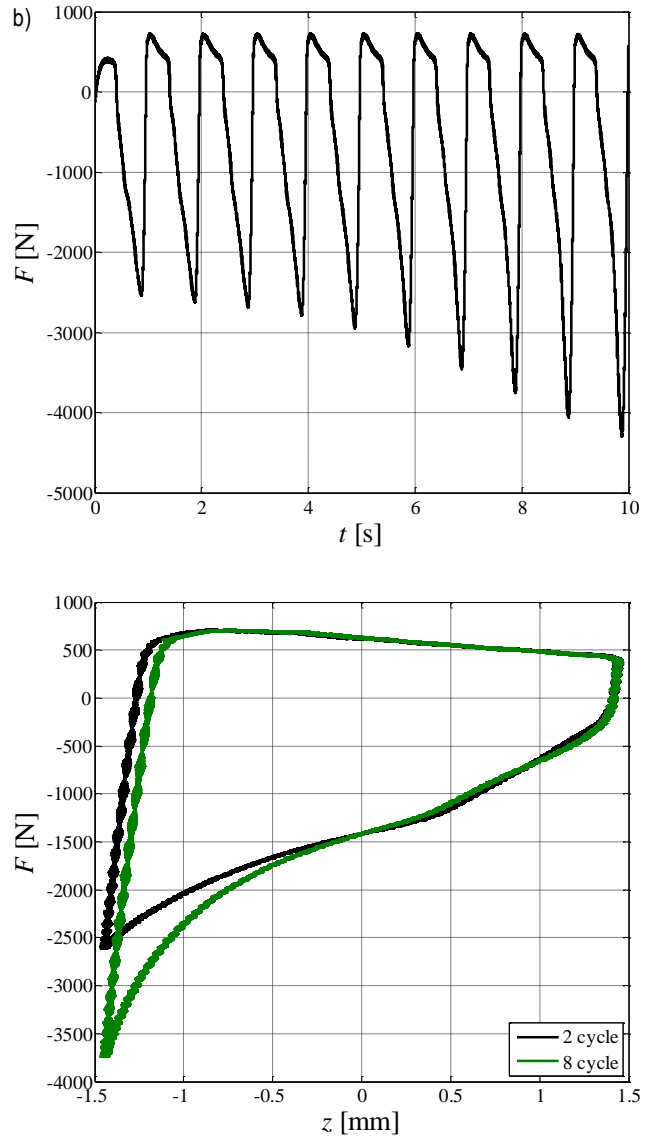

Fig. 8. Force vs. time and force vs. piston displacement; $A=1.44 \mathrm{~mm}$, $f=1 \mathrm{~Hz}$ : a) $l=1.0 \mathrm{~A}$, b) $l=3.0 \mathrm{~A}$
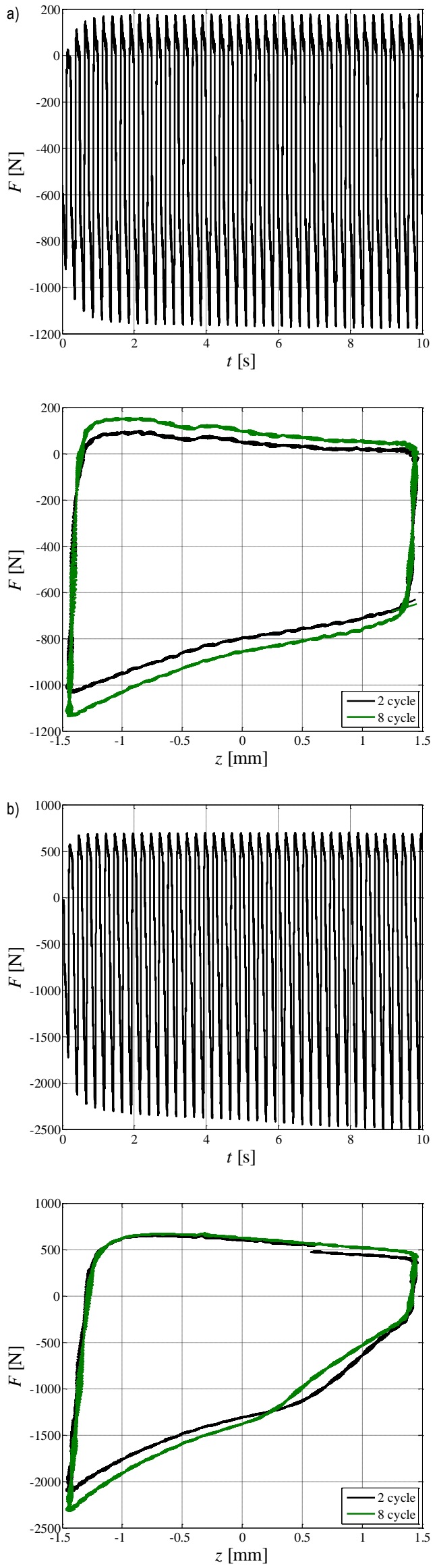

Fig. 9. Force vs. time and force vs. piston displacement; $A=1.44 \mathrm{~mm}$, $f=4 \mathrm{~Hz}$ : a) $I=1.0 \mathrm{~A}$, b) $l=3.0 \mathrm{~A}$ 

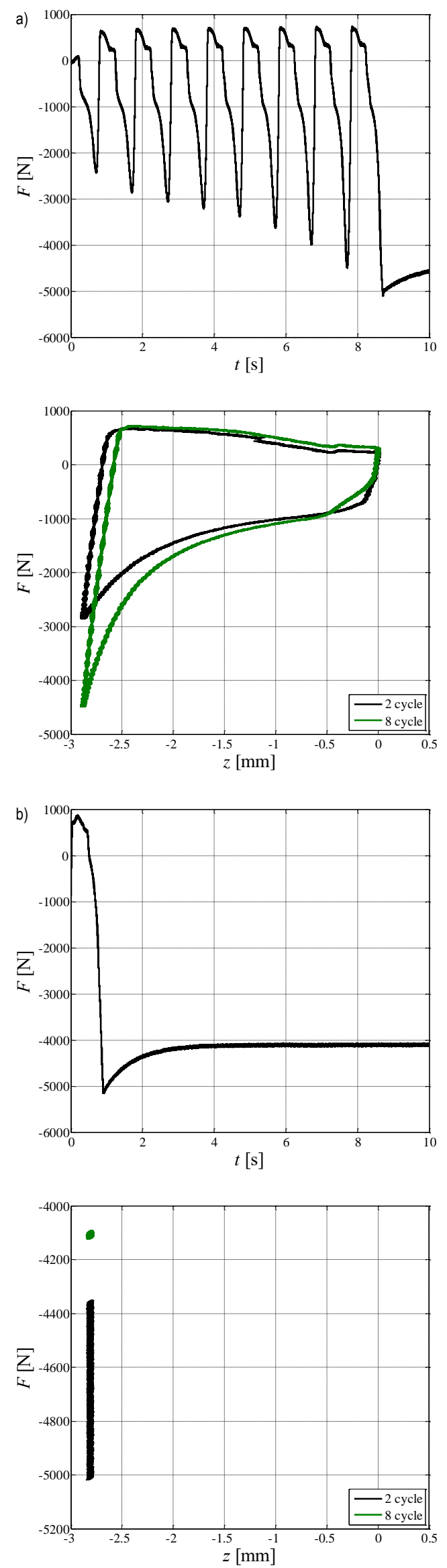

Fig. 10. Force time history and force vs. piston displacements; $A=1.44 \mathrm{~mm}, f=1 \mathrm{~Hz}, x_{0}=1.44 \mathrm{~mm}$ : a) $l=1.0 \mathrm{~A}$, b) $l=3.0 \mathrm{~A}$
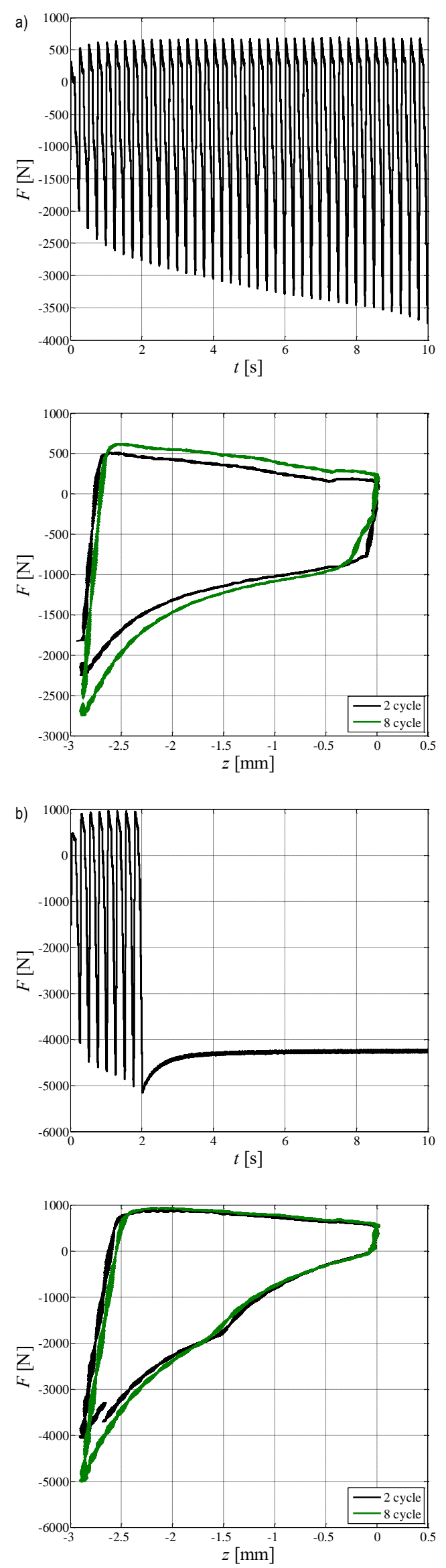

Fig. 11. Force time history and force vs. piston displacements; $A=1.44 \mathrm{~mm}, f=4 \mathrm{~Hz}, x_{0}=1.44 \mathrm{~mm}$ : a) $l=1.0 \mathrm{~A}$, b) $l=3 \mathrm{~A}$ 


\section{SUMMARY}

The paper summarises the results of quasi-static experimental testing of an MRSQD, with the focus on MR fluid clumping behaviour in the working gap. The influence of displacement inputs' parameters (amplitude and frequency), current level in the control coil and piston position offset on the damping force output of the device was also investigated. The acquired data are presented in the form of damping force time histories and damping force-piston displacements loops.

The discussion of the results lead us to the following conclusions:

- plots of damping force time histories and damping force-piston displacement loops are not symmetrical,

- the influence of current in the control coil is signifficant, the higher the current level, the higher the damping force,

- the influence of piston velocity (amplitude and/or frequency of displacement input) and similarly piston position offset on the damping force is rather small,

- in each test a significant increase of the damping force is observed with each cycle of the piston movement, which is associated with the clumping behavior of MR fluid in the gap,

Further research efforts will be focused on investigation the influence of magnetic signal dither to reducing the clumping effect in the device.

\section{REFERENCES}

1. Farjoud A., Ahmadian M., Cavey R. (2009a), Rheometer characterization of MR fluids in squeeze mode, Proc. SPIE 7288, Active and Passive Smart Structures and Integrated Systems, 72880R.

2. Farjoud A., Cavey R., Mehdi A., Craft M., (2009b), Magnetorheological fluid behavior in squeeze mode, Smart Materials and Structures, 18, 095001.

3. Farjoud A., Craft M., Burke W., Ahmadian M. (2011a), Experimental investigation of MR squeeze mounts. Journal of Intelligent Material Systems and Structures, 22, 1645-1652.
4. Farjoud A., Ahmadian M., Mahmoodi N., Zhang X., Craft M. (2011b), Non-linear modeling and testing of magneto-rheological fluids in low shear rate squeezing flows, Journal of Smart Materials and Structures, 20, 085013 .

5. Gołdasz J., Sapiński B. (2011), Model of a squeeze mode magnetorheological mount. Solid State Phenomena,177, 116-124.

6. Gong X., Ruan X., Shouhu X., Yan, Q., Deng H. (2014), Magnetorheological Damper Working in Squeeze Mode, Hindawi Publishing Corporation, Advances in Mechanical Engineering, 410158.

7. Guo Ch., Xinglong Gong X., Xuan S., Qin L., Yan Q. (2013), Compression behaviors of magnetorheological fluids under nonuniform magnetic field, Rheol. Acta, 52, 165-176.

8. Horak W. (2013), Theoretical and experimental analysis of magnetorheological fluid squeeze flow mode, PhD Thesis, AGH University of Science and Technology, Krakow.

9. Jolly M., R., Carlson J. D. (1996), Controllable squeeze film damping using magnetorheological fluids, Proc. of the 5th International Conference on New Actuators, Bremen, 333-336.

10. Kieburg Ch. (2010), MR Fluid Basonetic 4035, BASF Technical Information.

11. Kim J.-H. (2014), Damping force control filled with magnetorheological fluids and engine mount having the same, US Patent No. US 8,672,105 B2.

12. Manecke P, Winkler B., Manhartsgruber B. (2006), Magnetorheological damper, US Patent 7070027.

13. Mazlan S.A., Ekreem N. B., Olabi A.G. (2007, The performance of magnetorheological fluid in squeeze mode, Smart Materials and Structures, 16, 1678-1682.

14. Mazlan S.A (2008), The behaviour of magnetorheological fluids in squeeze mode, Ph.D. Dissertation, University of Dublin.

15. Sapiński B., Gołdasz J. (2015), Development and performance evaluation of an MR damper in squeeze mode, Smart Materials and Structures, 24, 115007.

16. Zhang X. J., Farjud A., Ahmadian M., Guo K. H., Craft M. (2011), Dynamic Testing and Modelling of an MR Squezee Mount, Journal of Intelligent Material Systems and Structures, 22, 1717-1728.

This work is supported by AGH University of Science and Technology under research program No. 11.11.130.958 